\title{
Cerebral Gas Embolism from Bronchoscopic Argon Plasma Coagulation: A Case Report
}

\author{
Yasmeen Shaw Ken Y. Yoneda Andrew L. Chan \\ Division of Pulmonary, Critical Care and Sleep Medicine, University of California, Davis Medical Center and \\ Veterans Affairs Northern California Health Care System, Sacramento, Calif., USA
}

\section{Established Facts}

- Argon plasma coagulation is relatively safe and effective in the management of obstructing lesions and hemorrhage in the central airways.

- However, argon plasma coagulation has been reported to be complicated by systemic gas embolism resulting in cardiodynamic collapse.

\section{Novel Insights}

- The position of the patient at the time of bronchoscopy might contribute to the location of systemic emboli.

- The proximity of the pulmonary venous circulation to the distal bronchi may represent a conduit for systemic emboli.

\section{Key Words}

Argon plasma coagulation • Bronchoscopy • Complication • Cerebral gas embolism

\section{Abstract}

Argon plasma coagulation (APC) is a common and safe bronchoscopic technique used in the management of obstructing lesions and hemorrhage in the central airways. Compli- cations of bronchoscopic APC are uncommon and include hemorrhage, perforation and fire in the airways. While bronchoscopic APC has been reported to cause systemic gas embolization and associated cardiovascular collapse, we report a case of cerebral gas embolization that occurred during bronchoscopic APC and highlight underappreciated potential risk factors for its occurrence.

Copyright $\odot 2011$ S. Karger AG, Basel

\section{KARGER}

Fax +41 613061234 E-Mail karger@karger.ch www.karger.com
(C) 2011 S. Karger AG, Base 0025-7931/12/0833-0267\$38.00/0

Accessible online at: www.karger.com/res
Yasmeen Shaw

4150 V Street, Suite 3400

Sacramento, CA 95817 (USA)

Tel. +1 9167343643

E-Mail yasmeen.shaw@ucdmc.ucdavis.edu 


\section{Introduction}

Bronchoscopy is considered a safe procedure [1]. In addition, argon plasma coagulation (APC) performed via flexible bronchoscopy is a safe and effective method for the management of airway obstruction [2]. It involves non-contact electrocoagulation, utilizing electrically conductive argon plasma to deliver high-frequency current to the target area [2]. With this technique, argon plas$\mathrm{ma}$ is delivered from the tip of the flexible probe at flows of 0.3-2 liters/min [3]. Some experts favor APC over neodymium:yttrium aluminum garnet (Nd:YAG) laser therapy for the purpose of endobronchial coagulation and vaporization due to its superior safety profile, lower cost and ease of use [4]. Nevertheless, while both of these thermal modalities have established track records of safety, serious complications such as systemic gas embolization have been reported in association with their use [3, $5,6]$. Bronchoscopically delivered APC has been specifically reported to be complicated by systemic gas embolism to the left ventricle and cardiodynamic collapse requiring cardiopulmonary resuscitation $[3,7]$. An extensive review of the published English-language literature yielded no reports of cerebral gas embolism due to bronchoscopic APC use. However, in the German literature, Osseiran et al. [8] published a case of arterial gas embolism with central nervous system effects that occurred during endobronchial APC. We present a case of cerebral arterial gas embolism occurring during bronchoscopic APC used in the treatment of an endobronchial tumor.

\section{Case Report}

An 88-year-old female, never-smoker, was evaluated for a repeat bronchoscopic thermal ablation procedure to treat the recurrence of a solitary right upper lobe (RUL) endobronchial squamous papilloma. Approximately 18 months prior, she had developed a cough, and a chest radiograph demonstrated a loculated hydropneumothorax in the right apex. Bronchoscopy at that time revealed an endobronchial tumor emanating from the anterior segment of the RUL bronchus completely obstructing the RUL orifice. Following APC and cryoprobe therapy, the RUL orifice and the posterior and apical segments were free of tumor, with a residual tumor occluding the anterior segment. Her cough abated and a follow-up chest radiograph revealed resolution of the hydropneumothorax. When her cough and hydropneumothorax recurred, she was referred back for the repeat interventional procedure (fig. 1).

Flexible bronchoscopy, with the patient in a semi-recumbent position, revealed a tumor completely occluding the RUL bronchus. Using a non-contact technique, APC at $30 \mathrm{~W}$ and a gas flow of 1 liter/min was applied to the tumor, followed by blunt debride-

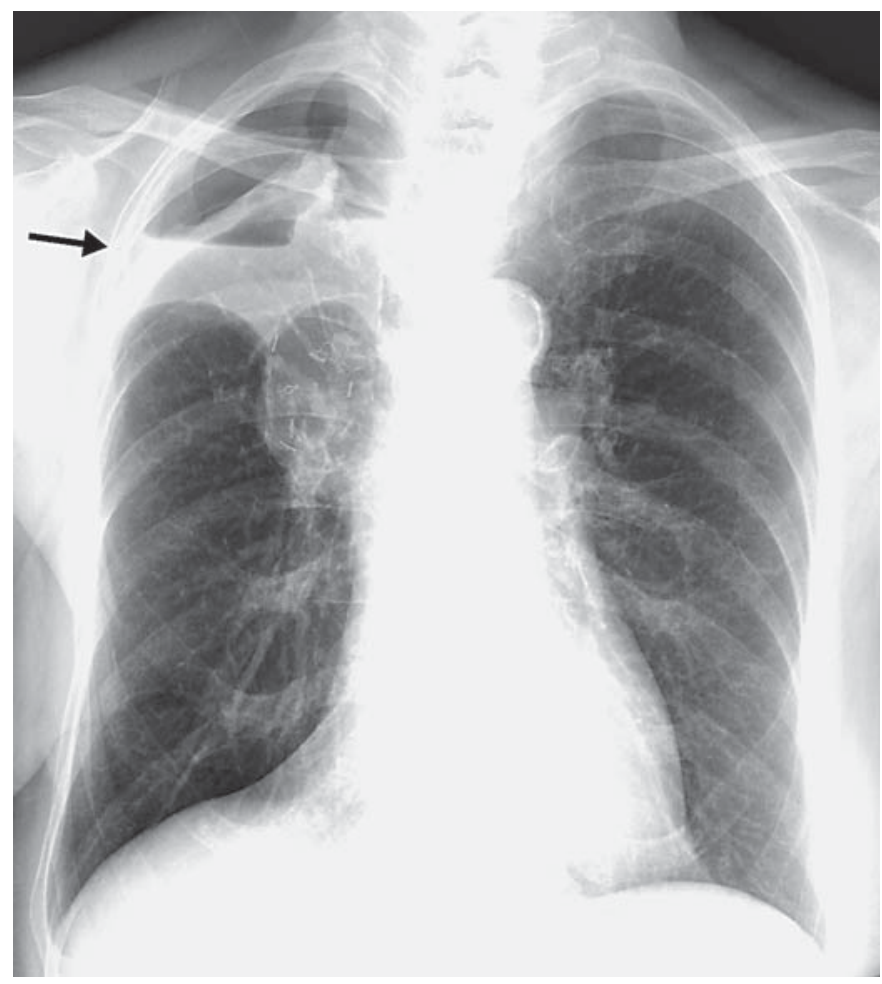

Fig. 1. Posterior-anterior view chest radiograph demonstrating a right apical hydropneumothorax (arrow).

ment with suction and biopsy forceps. This procedure resulted in patency of the RUL apical and posterior segments; however, the anterior segment of the RUL again remained occluded by the tumor. Brisk bleeding emanating from the anterior segment of the RUL required continued utilization of bronchoscopic APC to achieve hemostasis. Significant blood loss, estimated at $100 \mathrm{ml}$, was noted.

Towards the end of the APC procedure, the patient suddenly became apneic-bradycardic with a nadir heart rate of 32 beats/ min and hypertensive. Her level of consciousness decreased and she became unresponsive to deep stimuli. The patient was emergently resuscitated, endotracheally intubated, mechanically ventilated with an $\mathrm{FiO}_{2}$ of 1.0 , and was placed temporarily in a Trendelenburg position. Cardiac enzymes, renal function, blood sugar and electrolytes were within normal limits. Head computed tomography revealed multiple bilateral punctuate foci of very low attenuation in the subcortical white matter, likely representing gas. No acute transcortical infarction was noted at that time (fig. 2). Generalized tonic-clonic seizures occurred $5 \mathrm{~h}$ after bronchoscopy and were subsequently controlled with benzodiazepines and additional anti-epileptic medications. Seizure activity was confirmed by electroencephalogram, and repeat electroencephalogram following initiation of anti-epileptic drugs revealed resolution of the seizures. Magnetic resonance imaging of the head showed multiple areas of restricted cortical diffusion consistent with end-arterial infarcts, including bilateral thalamic infarcts. Magnetic resonance angiogram of the brain and neck re- 


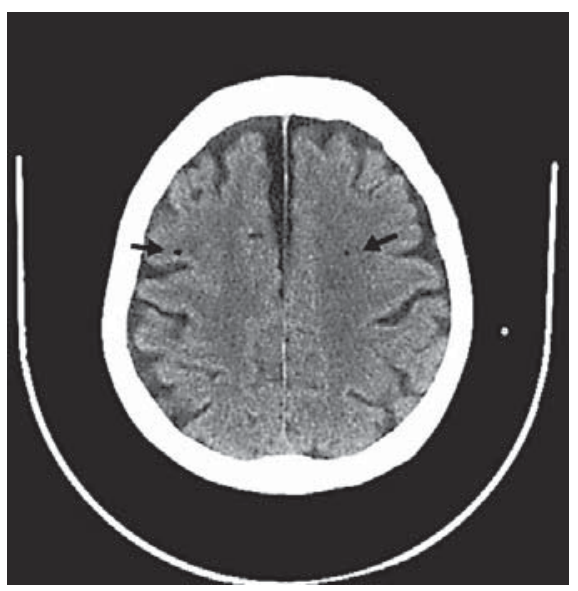

Fig. 2. Head computed tomography without contrast showing several punctuate foci of very low attenuation (arrows) in subcortical white matter bilaterally, most likely representing gas without acute transcortical infarction.

vealed no stenosis or aneurysmal dilatation of the vessels. Transfer to a facility offering hyperbaric oxygen therapy was attempted, but could not be logistically accommodated. Given the patient's rapid progression to severe neurologic injury, echocardiography was not performed as it would offer little therapeutic benefit. The patient expired a few days later after the family decided to withdraw support in light of the patient's grim prognosis for any meaningful neurological recovery.

\section{Discussion}

Bronchoscopic APC in this patient was associated with the sudden development of apnea, bradycardia, hypertension and an altered level of consciousness. This clinical deterioration, associated with generalized seizures, was most likely due to the development of cerebral gas emboli causing multiple end-arterial acute infarcts in the cortex and thalami. Thromboembolic and hemorrhagic etiologies of stroke were excluded given the findings of air emboli on computed tomography of the head and normal vasculature on magnetic resonance angiogram. The exact mechanisms responsible for the development of cerebral gas embolism in this case are not known, but several have been proposed for the development of systemic gas embolism during bronchoscopic laser and APC treatment: (1) high-pressure cooling or argon gas enters bronchus-pulmonary venous fistulas created by coagulation or malignant invasion of tissue; (2) gas enters the pulmonary arterial system and subsequently the systemic circulation via an intracardiac shunt; (3) gas trapped under high pressure due to occlusion of a bronchus by the bronchoscope is forced into vascular channels, and (4) gas is forced into bronchovascular channels by positive pressure ventilation $[3,6]$. Similar arguments concerning possible etiologies of cerebral air embolism, this time following uneventful flexible bronchoscopy, have been made by Azzola et al. [9].

Although an echocardiogram was not performed at the time of acute clinical deterioration in our patient, it is likely that gas bubbles would have been visualized in the left ventricular space, as has been reported in the case series by Reddy et al. [3]. Gas emboli could have entered the systemic circulation via a patent foramen ovale, but this possibility was not investigated in our patient in light of her progressive neurological deterioration and subsequent demise. Reddy et al. [3] suggest that systemic argon gas embolization may occur in a similar fashion to that proposed for Nd:YAG laser gas embolization [5]. Argon gas, exiting the tip of the flexible probe under low flow rates, but relatively high pressures, crosses mucosal membranes into blood vessels via bronchovascular fistulae that are created by coagulation of the involved tissue. Indeed, animal studies using a sheep model by Feller-Kopman et al. [5] found a positive correlation between the rate of gas flow and the presence of gas emboli after thermal ablation therapy in the airways. The authors showed that thermal ablation therapy without gas flow did not result in gas emboli in the sheep model. Interestingly, APC therapy in the trachea was associated more commonly with gas embolization to the right atrium likely via systemic veins. The opposite was true for APC ablation to the bronchi, where gas embolization occurred to the left atrium likely via the pulmonary veins, a possible explanation for the systemic gas embolization in our patient. In our case, positive pressure ventilation was not used and is therefore not a consideration in this case.

Our case highlights some potentially interesting facets to systemic gas embolism. Of note, in all 3 cases reported by Reddy et al. [3, 7], APC was used to terminate endobronchial bleeding, as was the case for our patient. Bleeding may represent an airway-pulmonary venous communication, affording a direct conduit for cooling or argon gas to enter into the left heart. Therefore, it is possible that active airway bleeding represents a significant risk factor for systemic gas embolism. Additionally, it is possible that superheated blood simply released dissolved gases into the same circulation, rather than cooling or argon gas being forced into the pulmonary venous circulation. Of note, in our case and in the 3 cases by Reddy et al. [3], APC was applied distal to a main bronchus. The anatomy of 
the pulmonary venous and pulmonary arterial circulation is not parallel. The pulmonary arterial circulation tracks along side the airways, while the pulmonary venous circulation comes into proximity with the airways intermittently at points distal to the main bronchus and bronchus intermedius on the right and the mid to distal main bronchus on the left. In the RUL, it is adjacent to the anterior segment, the exact area where we encountered brisk bleeding and at which the APC was directed. This anatomic difference may also account for the differences in left- and right-sided emboli from APC applied to the bronchi and trachea, respectively, as reported by FellerKopman et al. [5]. These possibilities appear to be distinct from, but are consistent with, postulates by Feller-Kopman and colleagues $[3,7]$. Whether the patient's RUL hydropneumothorax played a part in the development of systemic gas emboli is uncertain, but is considered less likely due to the lack of significant tension characteristics on the chest radiograph.

Systemic gas embolism following bronchoscopic APC usually connotes a potentially fatal outcome $[10,11]$, as was ultimately the case in our patient. The semi-recumbent position of the patient at the time of bronchoscopy possibly enabled the systemic embolization of gas to the cerebral circulation. A supine position may have emphasized cardiac complications instead, due to the relative positioning of cardiac chambers in non-dependent positions [10]. Nevertheless, after gas embolization occurred, the patient was resuscitated and placed on $100 \%$ oxygen in an attempt to reduce the size of the gas emboli by increasing the gradient for diffusion of the gas into the bloodstream [10]. The patient was initially placed in a Trendelenburg position, but was subsequently placed supine because of concern about aggravating possible cere- bral edema. Once gas bubbles were noted to be present in cerebral small arteries, pathologic damage had likely occurred due to a reduction in perfusion distal to the obstruction and to inflammation caused by the bubbles themselves [10]. Corticosteroids to combat cytotoxic edema from cerebral arterial gas embolism were not administered because of the lack of proven efficacy and the possibility that corticosteroids themselves may aggravate neuronal ischemic injury. However, hyperbaric oxygen therapy, either immediate or delayed, may have provided some benefit, although it was logistically impossible to achieve this for the patient at that time. Its benefit may have included a reduction in the size of the gas bubble by raising ambient pressure and systemic hyperoxia, greatly enhancing diffusion gradients. Cerebral edema may have been ameliorated by reducing blood vessel permeability and enhancing blood-brain barrier integrity.

\section{Conclusion}

We report the first case of cerebral systemic gas embolization from bronchoscopic APC published in the English-language literature. Systemic gas embolism, albeit rare, is associated with gas flow from the tip of the flexible probe together with tissue contact. Utilizing a noncontact technique and minimizing gas flow rates may help to prevent this devastating complication. Active airway bleeding during bronchoscopic APC and its application to selected areas distal to the main bronchus on the right or the mid to distal main bronchus on the left may represent risk factors for the development of systemic gas emboli.

\section{References}

1 Jin F, Mu D, Chu D, Fu E, Xie Y, Liu T: Severe complications of bronchoscopy. Respiration 2008;76:429-433.

-2 Morice RC, Ece T, Ece F, Keus L: Endobronchial argon plasma coagulation for treatment of hemoptysis and neoplastic airway obstruction. Chest 2001;119:781-787.

$\checkmark 3$ Reddy C, Majid A, Michaud G, Feller-Kopman D, Eberhardt R, Herth F, Ernst A: Gas embolism following bronchoscopic argon plasma coagulation: a case series. Chest 2008;134:1066-1069.

4 Wahidi MM, Herth FJ, Ernst A: State of the art: interventional pulmonology. Chest 2007;131:261-274.
5 Feller-Kopman D, Lukanich JM, Shapira G, Kolodny U, Schori B, Edenfield H, Temelkuran B, Ernst A, Schindel Y, Fink Y, Fox J, Bueno R: Gas flow during bronchoscopic ablation therapy causes gas emboli to the heart: a comparative animal study. Chest 2008; 133 : 892-896.

6 Tellides G, Ugurlu BS, Kim RW, Hammond GL: Pathogenesis of systemic air embolism during bronchoscopic Nd:YAG laser operations. Ann Thorac Surg 1998;65:930-934.

7 Goldman YE, Panzica PJ, Pinto DS, Delatorre R, Feller-Kopman D, Ernst A: Cardiac arrest due to left ventricular gas embolism after bronchoscopic argon plasma coagulation: a case report. J Bronchol 2007;14:33-35.
-8 Osseiran K, Barchfeld T, Dellweg D, Haidl P: Cerebral arterial gas embolism as complication during the therapeutic endobronchial use of argon plasma coagulation. Pneumologie 2008;62:353-354

-9 Azzola A, von Garnier C, Chhajed PN, Schirp U, Tamm M: Fatal cerebral air embolism following uneventful flexible bronchoscopy. Respiration 2010;80:569-572.

10 Muth CM, Shank ES: Gas embolism. N Engl J Med 2000;342:476-482.

11 Van Liew HD, Conkin J, Burkard ME: The oxygen window and decompression bubbles: estimates and significance. Aviat Space Environ Med 1993;64:859-865. 